\title{
CONTINENTAL DIVISION OF LOAD AND BALANCED ANT FAMILY (BAF) ALGORITHM FOR LOAD BALANCING ON PUBLIC CLOUD
}

\author{
Anisaara Nadaph and Prof. Vikas Maral \\ Department of Computer Engineering, K.J College of Engineering and Management \\ Research Pune
}

\begin{abstract}
Increasing usability of internet is creating huge data which should be managed by the industries, but this should not affect the processing time which may create inconvenience to end user. As cloud is emerging as back bone of IT industry there are much enhancement needed in it Many companies are switching their data from small storage location to Cloud, The migration is done such that the companies do not have a burden to purchase the Infrastructure they merely have to rent out the Infrastructure but the migration should not cost on the speed of storage or retrieval of the data from the server. Load balancing is one of the major issue in cloud computing, but these problems are Tractable There are many algorithm for load balancing which has advantage over the other in this paper Ant colony algorithm is studied.
\end{abstract}

\section{KEYWORDS}

Balanced Ant family algorithm, cloud computing, continent, load balancing

\section{INTRODUCTION}

Load balancing is a technique in which all server are utilized in an optimal manner such that none of the server remain Idle when other server are overloaded, and if optimal balancing of load is not done the end user may find delay in the speed. There are different search engine working for better load balancing techniques. Google has a Compute Engine that provides server-side load balancing so you can distribute incoming network traffic across multiple virtual machine instances. Load balancing provides the following benefits with either network load balancing or HTTP load balancing:

- Scale your application

- Support heavy traffic

- Detect unhealthy nodes

- Balance loads across regions

- Divert job to the closest node machine

- Support content-based routing

As the type of job is unpredictable i:e the job are dynamic. Different types of jobs are static or dynamic, in static job type the current state of job is immaterial whereas in dynamic job strategy the current state is of concern. There are different load balancing algorithm like Round Robin, Ant colony, Central load balancing. NIST definition[9] of cloud computing is " model for enabling omnipresent, handy, on- order access to a shared group of computing resources which

DOI: $10.5121 /$ ijci.2014.3504 
International Journal on Cybernetics \& Informatics (IJCI) Vol. 3, No. 5, October 2014

can be rapidly released with minimal organization exertion or service providers dealings this fact has given a direction to shift organization from companies self-Infrastructure to cloud[2][3][6][8].

\section{DIFFERENT ALGORITHM STUDIED}

This section gives the brief study of different algorithm that are applied for balancing of load on the server

\subsection{ROUND ROBIN}

In this each server is assigned job one at time and then again the first server.
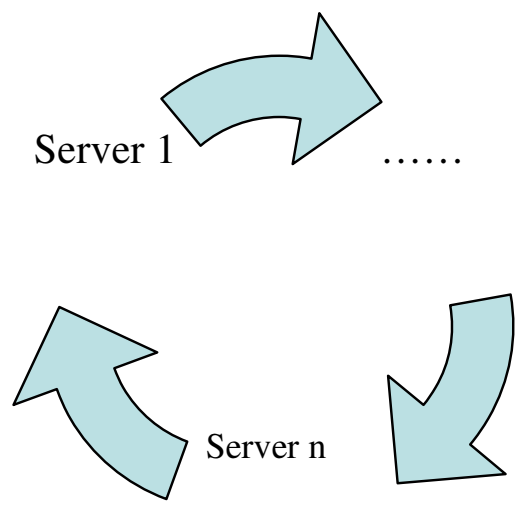

Figure 1: Round robin system

\subsection{HONEY BEE BEHAVIOR Algorithm[4]}

In this the natural behaviour of the Honey bees are considered, There are different types of bees in the honey comb, from which the scout bee go about in search of food and comes back home and perform waggle dance on the provided dance floor, This strategy is applied for balancing of task on cloud.

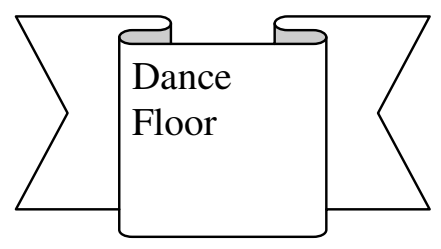

Figure 2: Honey comb with dance floor

\subsection{Central Load Balancing [1]}

In this strategy balances load for the virtual machine such that there is even load distribution for the distributed virtual machine 
International Journal on Cybernetics \& Informatics (IJCI) Vol. 3, No. 5, October 2014

\section{APPROACH APPLIED}

\subsection{Partitioning Technique}

As there are partitioning of the ip address based on region, the balancers are partitioned based on regions. There are 5 regions of the world.

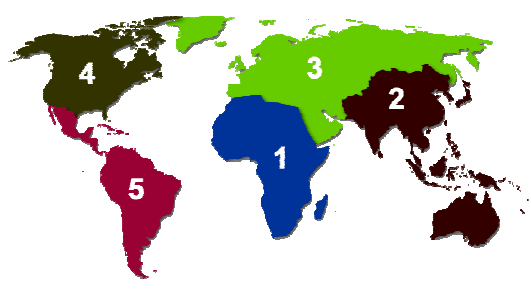

1. Africa

2. Asia

3. Europe

4. North America

5. South America

Hence there will be 5 balancer as per the region and the load of each balancer are checked and then the Balanced Ant Family algorithm (BAF) is applied to balance the load

\subsection{BALANCED ANT FAMILY ALGORITHM (BAF)[7][10]}

based on natural behaviour of Ant in which first the Ant moves in random direction and then find the shortest path for food. On the similar basis we will design an Ant family optimization algorithm. In this the Ant continuously update the result table, the algorithm will help for the optimal utilization of the resources in this global pheromone updation concept is given priority. The Ant task in the algorithm is to distribute load in even manner such that none of the server remain underloaded when other server is overloaded.The Ant are originated from the balancer node which is the regional balancer node (RBN) as per our system. The time when an Ant originate for foraging will be based on number of server in the network, As there can be many ant which can be originated from the regional balancer node(RBN) hence we will set a life timer(LT) for each ant, the LT is set such that too many ant may not be generated as it will create burden of maintaining information of each ant which may reduce the network efficiency.

If there are multiple food source which are at same distance the first food source is randomly selected by ant, In this the nodes are selected randomly first if sufficient space is found for storage of job, node is selected and the pheromone table is updated else if the

\section{SySTEM ARCHITECTURE}

This section gives the keen study of how the system should work, In the system there is Regional controller(RC) which gathers all the mandatory information from the Regional balancer (RB) and from the RB the data is transferred to the data storage server(DSS) by applying the Ant Colony algorithm. 

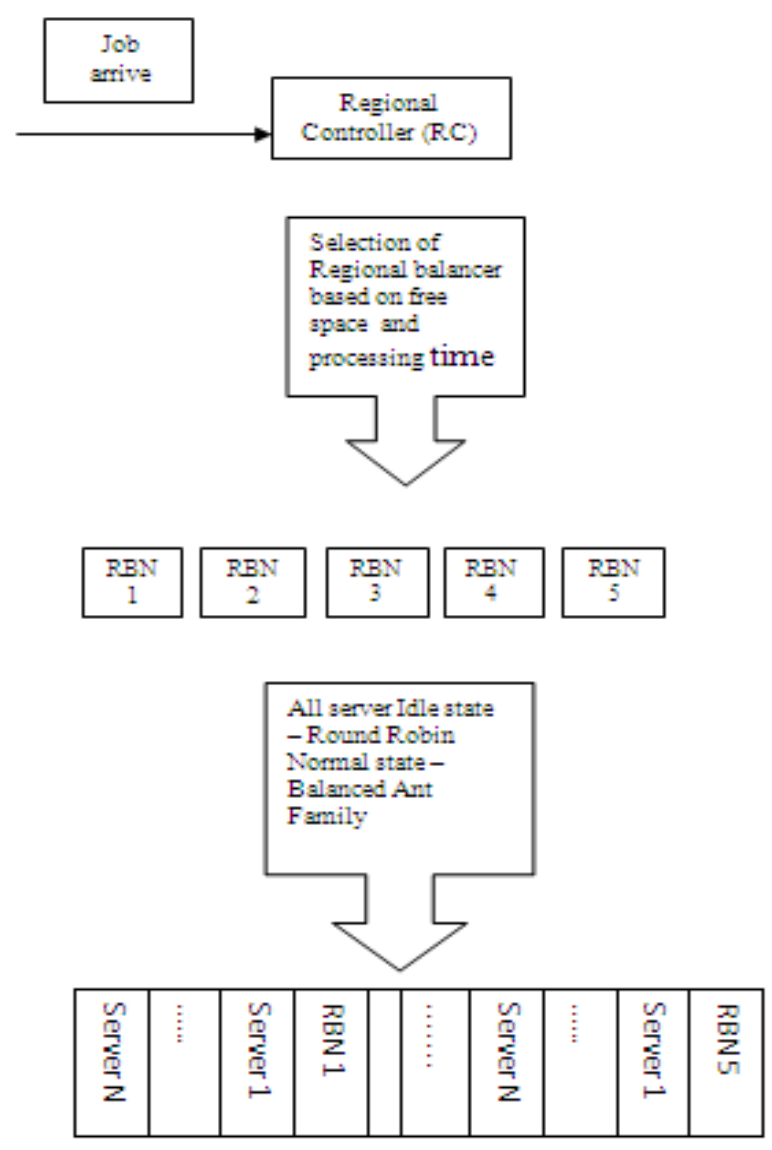

\section{AlgorithM APPLIED}

This section the detail study of different algorithm that are applied for the system

A. Alogrithm applied for selection of the Regional Balancer

1. Regional Controller $(\mathrm{RC})$ maintains the record of the regional balancer (RB)

2. Selection of the RB with maximum free space

B. Algorithm Applied for internal node selection

a. Idle state[5]

When all internal nodes are in Idle state then selection in done by Round Robin

b. Normal state (Balanced Ant Family algorithm)

1. Set the Threshold value for each node

2. Initially the Ant start movement from the regional balancer node (RBN).

3. Calculate the pheromone value to the nodes based on the processing time of the server. $\mathrm{L}=$ low processing time 
International Journal on Cybernetics \& Informatics (IJCI) Vol. 3, No. 5, October 2014

4. $\mathrm{H}=$ high

processing

time

Life Time(LT) for the ant starts

5. Ant maintain the result set table for the nodes,

6. Job arrives

7. Select the node randomly (target node $\{\mathrm{TN}\}$ )

8. If load[TN]>Threshold)

For all nodes $\mathrm{i}=1$ to $\mathrm{N}$

a. check the node with load[i]<Threshold and maximum free space assign the node as the destination node set loadflag $=1$

b. If loadflag $==0$ then check the pheromone content of the node, the node with $\mathrm{H}$ is assigned the value

9. If load[TN]<Threshold

Assign the job to the Node with maximum free space assign the node as the destination node set loadflag $=1$

\section{MATHEMATiCAL MODEL}

This section gives the module wise mathematical model of the system. For overall system uses divide and conquer strategy hence the model is written as

Decision of Regional Balancer Node (RBN). Job of different type will be input to the system, If a node is assigned for the storage of job the system will success otherwise the system fails. For all jobs. Let us assume there are $\mathrm{N}$ number of servers, as there are 5 regions the division will be N/5. Check the memory utilized by each Regional balancer and the RB with maximum free space should be considered

$$
\mathrm{F} 1=\mathrm{Mem}_{-} \mathrm{U} \_\mathrm{RB}(\mathrm{L})=\sum \mathrm{m}_{i=1} M i
$$

After the Regional balancer is selected for Idle state Round Robin which is the simplest is applied and When all nodes under the Regional balancer are in Normal state Ant Colony algorithm is applied, the PT(Processing Time) is obtained as

PT= Distance from the selected node/speed of the processor (2)

Time for processing $=$ Distance of server from randomly selected node by Speed of each processor.

\section{EXPERIMENTAL SETUP}

There are 8 nodes used for Balanced Ant Family(BAF) with 500GB of hard disk. One Main controller and a conceptual Regional balancer to keep record of the internal nodes. Different size of jobs can be considered for taking reading based on time in millisecond and space as which server will be used most as per the algorithm

\section{Conclusion}

As Cloud is acquiring huge demand, load balancing is of prime important to increase the response time of the job. The divide and conquer strategy applied seems efficient and different algorithm applied at different load time appears efficient The Processing Time required for the nodes are considered same for the experiment, as speed and distance are same for all the nodes a tunned result can be obtained by considering different speed processor 
International Journal on Cybernetics \& Informatics (IJCI) Vol. 3, No. 5, October 2014

\section{Future Work}

1. For deciding Life Time (LT) for ant a suitable algorithm need to be decide

2. The Reload Time(RT) of Central control need to be decided or there should be some algorithm to decide the RT

\section{REFERENCE}

[1] A. Bhadani and S. Chaudhary,"Performance evaluation of web servers using central load balancing policy over virtual machine on cloud", Proceeding of the Third Annual ACM Banglore Conference , January 2010

[2] Adler, Load balancing in the cloud: Tools, tips and techniques, http://www.rightscale. com/info center/white-papers/Load-Balancing-in-the-Cloud.pdf", 2012

[3] Bhadani A. and Chaudhary S., 3rd Annual ACM Bangalore Conference, 2010

[4] Babu L.D. a, P. VenkataKrishnab," Honey bee behavior inspired load balancing of tasks in cloud computing environments", www.elsevier.com/locate/asoc,2013.

[5] GaochaoXu, Junjie Pang, and Xiaodong Fu "A Load Balancing Model Based on Cloud Partitioning for the Public Cloud" IEEE TRANSACTIONS ON CLOUD COMPUTING YEAR 2013

[6] Google Trends, Cloud computing, "http://www.google. com/trends/exploreq=cloud computing", 2012.N. G. Shivaratri, P. Krueger, and M. Singhal, Load distributing for locally distributed systems, Computer, vol. 25, no. 12, pp. 33-44, Dec. 1992

[7] Kumar Nishant, Pratik Sharma, Vishal Krishna, Chhavi Gupta and KunwarPratap Singh, "Load Balancing of Nodes in Cloud Using Ant Colony Optimization", 2012 14th International Conference on Modelling and Simulation

[8] Microsoft Academic Research, Cloud computing, "http:// libra.msra.cn/Keyword/6051/cloudcomputing?query= cloud\%20computing", 2012.

[9] P.Mell and T. Grance, The NIST definition of cloud computing, "http://csrc.nist.gov/publications/nistpubs/800-145/SP800-145.pdf", 2012

[10] Ratan Mishra1 and AnantJaiswal "Ant colony Optimization:"A Solution of Load balancing in Cloud", International Journal of Web Semantic Technology Vol.3, No.2, April 2012. 